\title{
Two Dimensional Crystallization and Projection Structure of a Human Connexin26
}

\author{
A. Oshima*, K. Tani*, Y. Hiroaki*, Y. Fujiyoshi* and G.E. Sosinsky** \\ * Department of Biophysics, Faculty of Science, Kyoto University, Oiwake, Kitashirakawa, Sakyo- \\ ku, Kyoto 606-8502, Japan \\ ** National Center for Microscopy and Imaging Research, Department of Neurosciences, University \\ of California San Diego, La Jolla, CA 92093
}

Gap junctions (GJs) are membrane channels mediating direct intercellular communication between connected cells. Connexin26 (Cx26) is the second smallest and one of the most important members of the family of GJ proteins, the connexins, and is highly expressed in organs such as liver, skin, brain, kidney, intestine and inner ear. Mutations in the Cx26 sequence have been shown to be the cause of many cases of hereditary deafness and skin disease [1]. In addition, while Cx26 knockout mice are fertile and can breed, they cannot carry the fetus to term and the fetuses die in utero demonstrating that Cx26 plays a critical role in pregnancy and early embryonic development [2].

It is essential and indispensable to visualize the high resolution structure of Cx26 GJ in order to understand how this important membrane channel functions. We focused on studying the $\mathrm{Cx} 26$ structure because of its functional importance and because the short cytoplasmic tail may make it more amenable to crystallization. Here, we report the two-dimensional crystallization of a mutant human Cx26 (M34A) by reconstitution into lipid bilayers. This mutation site was discovered in cases of non-syndromic hereditary deafness [1]. We over-expressed a hexahistidine tagged human M34A Cx26 using baculovirus recombinant expression system. Detergent solubilized hemichannels, referred to as connexons, purified by $\mathrm{C}$-terminal $\mathrm{His}_{6}$ tag were reconstituted into the synthetic lipid dioleoylphosphatidylcholine (DOPC) using dialysis and temperature variations resulting in formation of stacked two dimensional membrane crystals (Fig.1A). Since images of negatively stained crystals suggested that these crystals were of varying thicknesses, we used thin section electron microscopy to examine cross-sections through pelleted crystals. These images showed that our crystals consist of multiple layers containing units of three bilayers (Fig.1B arrow) that often stack to form thick multilayer (Fig.1B arrowhead). It should be noted that the density of middle layer is higher than those of other flanking ones. For high resolution EM structure determination, these crystals were embedded in 1\% tannic acid, frozen into liquid nitrogen and examined by electron cryo-microscopy. After image processing, we consistently obtained information to $10 \AA$ resolution by electron crystallography (Fig.2A). Eleven images were processed and merged to reveal the projection map at $10 \AA$ resolution (Fig.2B). The orthorhombic unit cell parameters of crystals are $\mathrm{a}=\mathrm{b}=108.0 \AA, \gamma=90.0^{\circ}$. Projection maps contain highly hexameric channels similar to a previously published EM projection maps from isolated GJ p6 crystals of truncated Cx43 [3], except that a weak but significant density is observed in the channel pore. This inner density is off center from the vertical pore axis indicating that $\mathrm{Cx} 26$ channels have $\mathrm{p} 1$ rather than $\mathrm{p} 6$ crystallographic symmetry. In addition, adjacent molecules in the asymmetric unit have differing densities from each other suggesting a stagger between adjoining membrane layers. These initial projection maps indicate that this novel crystal form is amenable for a full $3 \mathrm{~d}$ structure determination of the Cx26 GJ channel. 
References

[1] R. Rabionet et al., Hum. Mutat. 16 (2000) 190.

[2] H.D. Gabriel et al., J. Cell Biol. 140 (1998) 1453.

[3] V.M. Unger et al., Nat. Struct. Biol. 4 (1997) 39.

[4] R. Henderson et al., Ultramicroscopy 19 (1986) 147.

[5] This work is supported by Grants-in Aid for SPR, Grant-in Aid for 21COE Kyoto University and JST (YF) and NIH grants GM065937-02(GS), RR04050 and NSF MCB-0131425(GS).



Figure 1 (A) Negatively stained electron micrograph of Cx26 2D crystal. (B) Thin section electron micrograph of Cx26 2D crystal. Arrow points to a three layered membrane unit of a crystal while the arrowhead points to a series of stacked crystals.

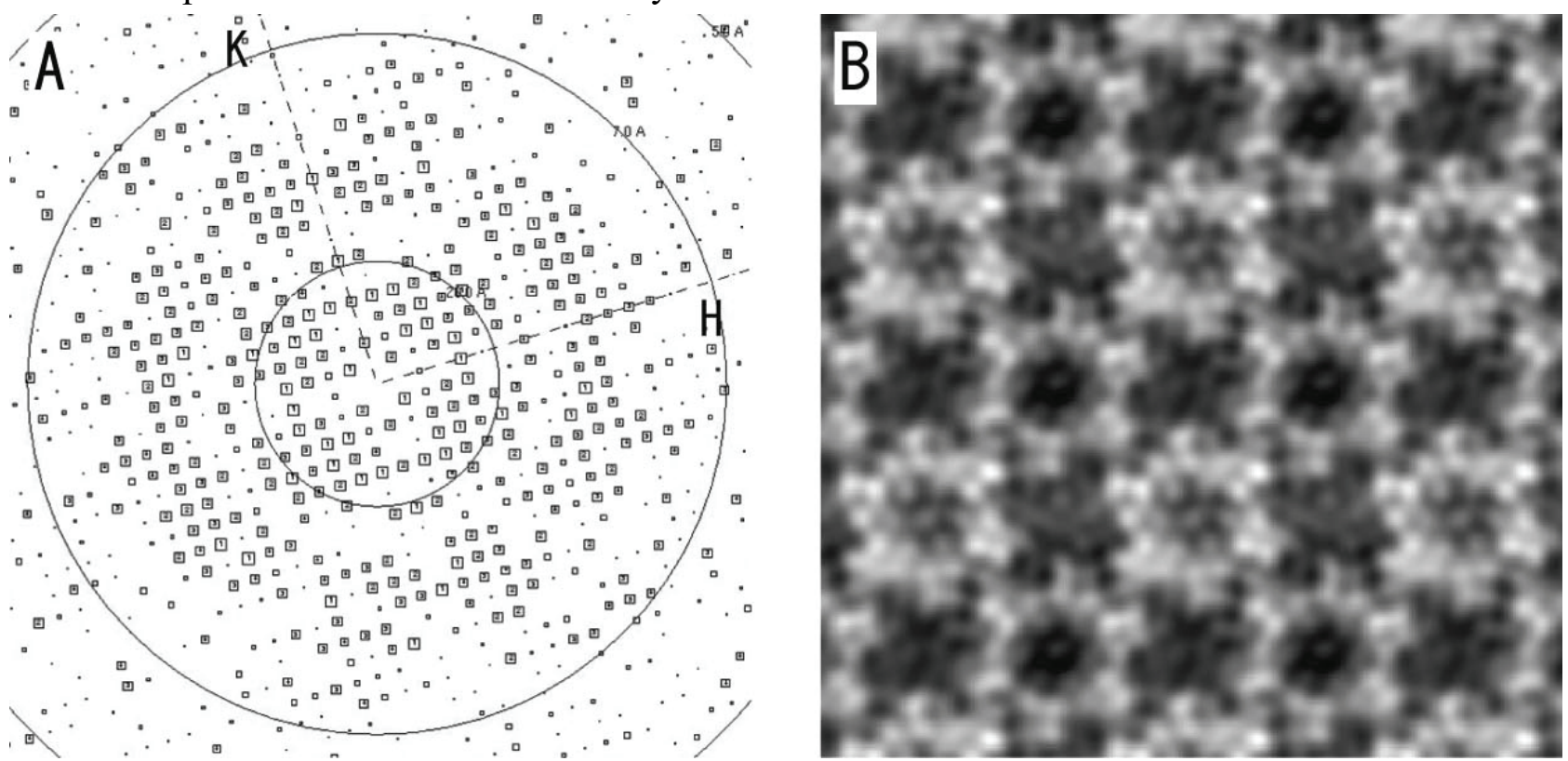

Figure 2 (A) Image-quality (IQ) plot [4] of a single image of frozen crystal. Each circle corresponds to following resolutions; inside $=20 \AA$, middle $=7 \AA$, outside $=5 \AA$. (B) $10 \AA$ resolution projection map calculated as $\mathrm{p} 1$ from 11 frozen crystal images. 